\title{
Spontaneous Unilateral Tubal Twin Ectopic Pregnancy: A Case-Based Discussion and Assessment
}

\author{
Vaneesha Vallabh-Patel, Robert Vera, Gustavo Martell, Sanja Kupesic Plavsic \\ Department of Obstetrics and Gynecology, Texas Tech University, EI Paso, Texas, USA
}

Corres pondence: Sanja Kupesic Plavsic, Professor, Department of Obstetrics and G ynecology, Paul L Foster School of Medicine Texas Tech University, 4800 Alameda Ave, EI Paso, Texas 79905, USA, P hone: 915-783-1700, e-mail: sanja.kupesic@ ttuhsc.edu

\section{ABSTRACT}

Introduction: Two percent of all 1st trimester pregnancies in the United States are ectopic. Transvaginal ultrasound is considered the mainstay in diagnosis. Color Doppler ultrasound can prove to be an added helpful modality in identifying ectopic pregnancies.

Case report: An 18-year-old nulliparous female presented with vaginal bleeding and lower abdominal pain. Pelvic exam revealed tenderness in the right adnexal region with elevated quantitative serum beta-hCG. A transvaginal ultrasound showed an echogenic ring adjacent to the right ovary and $\mathrm{M}$-mode and color Doppler revealed two fetal heart tones. Color Doppler enabled visualization of randomly dispersed tubal arteries and heart activity of both embryos. A right salpingectomy was performed without complications and beta hCG levels returned to undetectable levels.

Conclusion: B mode ultrasound is a vital diagnostic tool in the detection of ectopic pregnancy. Color Doppler provides both morphologic and functional information essential for determining the optimal therapeutic strategy. Three-dimensional ultrasound with color and/or power Doppler has proven us eful in both the initial diagnosis and follow-up of ectopic pregnancies especially in patients following assisted reproduction treatment.

Keywords: Tubal ectopic pregnancy, Transvaginal ultrasound, Color Doppler ultrasound, Three-dimensional ultrasound, Serum beta-hCG

\section{INTRODUCTION}

A n ectopic pregnancy occurs when the blastocyst implants in a location other than the endometrial lining of the uterine cavity. This holds true whether the present ectopic pregnancy is a singleton or multigestational. This occurrence, however, has greatly increased since the introduction of assisted reproductive technology (ART) and tuboplasty. A RT has increased the rate of ectopic pregnancies due to the mechanism of the procedure; how ever, tuboplasty has a mechanism similar to STDs, where adhesions can form postsurgery along with scar tissue inhibiting peristalsis of the fallopian tube when egg is transferred from the ovary to the uterus.

Two percent of all 1st trimester pregnancies in the $U$ nited States are ectopic, and these account for six percent of all pregnancy-related deaths. ${ }^{1}$ This is a significant decrease compared with the twelve percent reported by the Centers of Disease Control for the time period of 1979 to 1986, but still similar to the number approximated in the 1992 Center of Disease Control 'C urrent Trends in Ectopic Pregnancy'.

When comparing the types of ectopic pregnancies, fallopian tube pathology is the most common, currently estimated at $95 \%$, followed by ovarian pregnancy (3\%), and finally abdominal, cervical and cornual location at $<1 \% .{ }^{2}$ A lthough rare, the incidence of twin ectopic pregnancies is estimated to occur in $1 / 125,000$ pregnancies, ${ }^{3}$ making unilateral twin tubal gestations $1 / 200$ ectopic pregnancies. ${ }^{4}$ This phenomenon was first described in 1891 by De $0 t^{5}{ }^{5}$
We present a rare case of a symptomatic patient with spontaneous unilateral twin tubal gestations, with cardiac motion detected via transvaginal and color D oppler ultrasound.

\section{CASE REPORT}

A $n$ 18-year-old nulliparous female presented to the emergency department with the chief complaint of vaginal bleeding and lower abdominal pain. She states that the onset of pain occurred one week ago and describes the pain as intermittent in nature, ranging from 6 to 10 on a 10-point pain scale. She denies any radiation of the pain and also denies having any previous episodes of similar pain.

The patient's history is significant for irregular menstrual cycles, with her last menstrual cycle being 2 months prior to evaluation. She states that she has been sexually active since the age of 15 years old but denies any history of known STDs. The patient also denies any history of active medical problems or prior surgeries. The patient denies the use of any medications including oral contraceptives.

On physical examination, the patient was afebrile and her vital signs were within normal limits. Abdominal palpation elicited tenderness in the right lower quadrant, with no rebound or guarding. Pelvic examination revealed no blood in the vaginal vault and a closed cervical os. Bimanual exam revealed a normal sized uterus, normal left adnexa and tenderness in the right adnexal region. L aboratory results show ed a quantitative serum beta-hCG value of $2810 \mathrm{mIU} / \mathrm{ml}$ and normal CBC. 


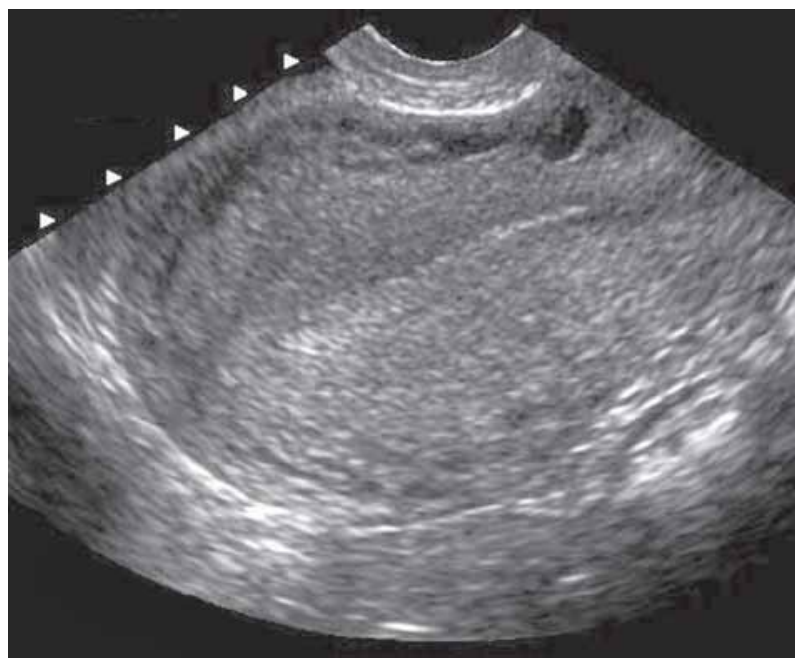

Fig. 1: Key to the diagnosis of ectopic pregnancy in determination of presence or absence of an intrauterine gestational sac correlated with quantitative serum beta-hCG levels. An ectopic pregnancy should be suspected, if transvaginal ultrasonography does not show an intrauterine gestational sac, when the beta-hCG level is higher than $1.500 \mathrm{mlU} / \mathrm{ml}$. In our patient, transvaginal ultrasound reveals hyperechogenic endometrium with no evidence of intrauterine pregnancy

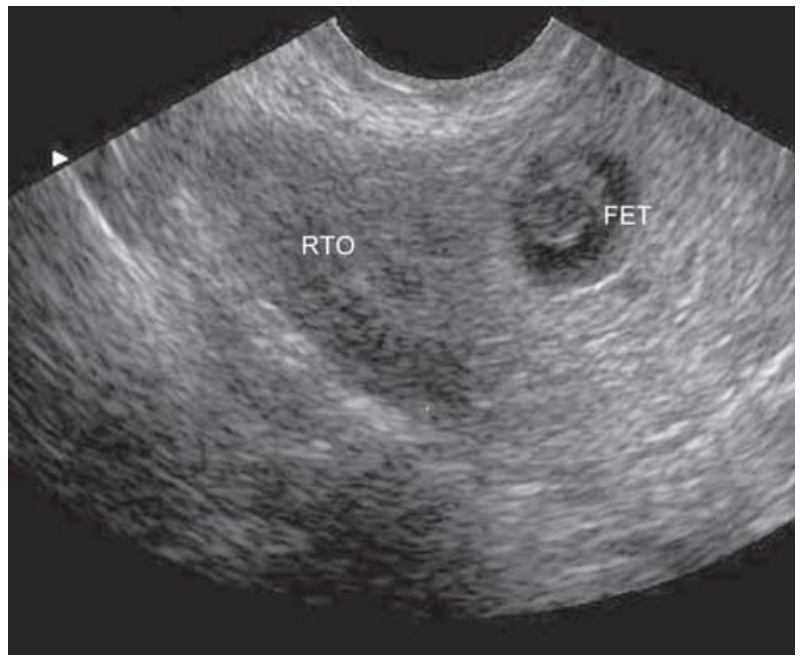

Fig. 2: The most common sonographic sign associated with ectopic pregnancy is an echogenic ring. This transvaginal ultrasound image illustrates a gestational sac with yolk sac and embryonic pole (FET) visualized adjacent to the right ovary (RTO)

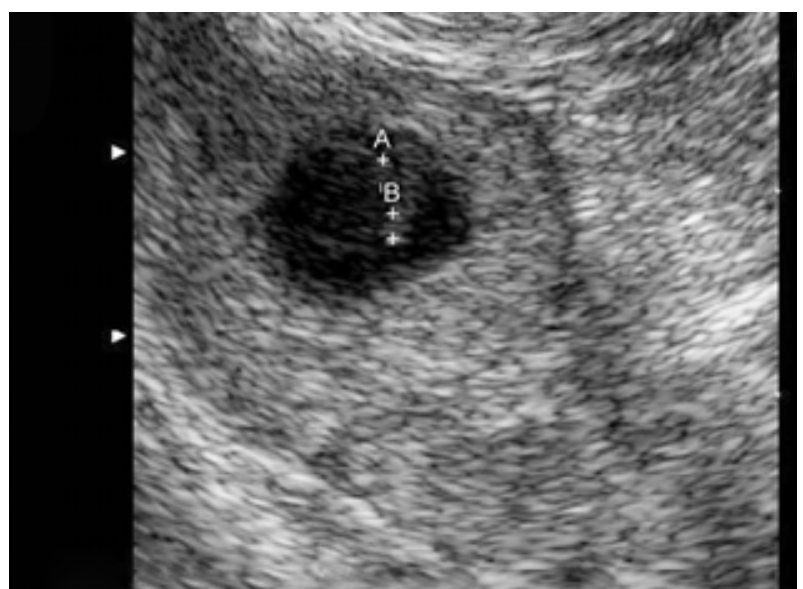

Fig. 3: Careful exploration of the gestational sac reveals two embryonic poles measuring $1 \mathrm{~mm}$ each. Sonographic finding is consistent with ectopic twin pregnancy

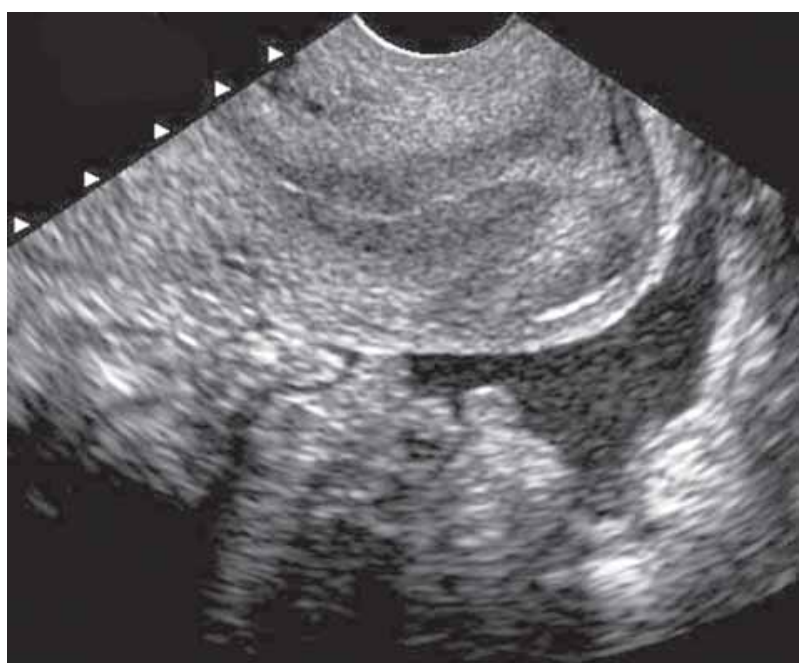

Fig. 4: Transvaginal ultrasound of free fluid in the posterior cul-de-sac

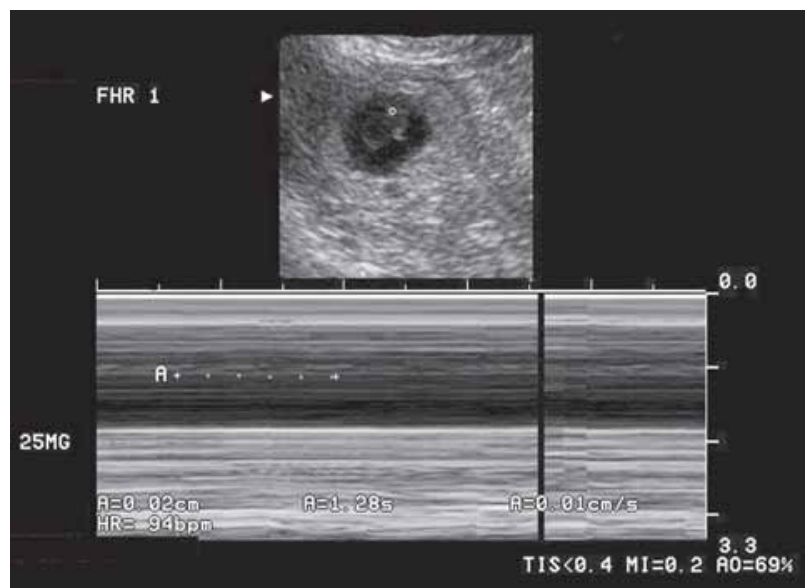

Fig. 5: M-mode ultrasound depicts heart activity of one of the embryos with heart rate of 94 beats per minute

A transvaginal ultrasound showed a uterus measuring $6.9 \times$ $4.2 \times 5.4 \mathrm{~cm}$. The endometrium was hyperechogenic, with no evidence of intrauterine pregnancy (Fig. 1). The right ovary measures $2.3 \times 1.5 \times 1.7 \mathrm{~cm}$. The left ovary measures $3.3 \times 1.9$ $\times 2.4 \mathrm{~cm}$. In the right adnexal region, adjacent to the ovary there was an echogenic ring measuring $1.5 \mathrm{~cm}$ in mean diameter (Fig. 2). Careful exploration of the gestational sac revealed two embryonic poles measuring $1 \mathrm{~mm}$ (Fig. 3). A small amount of free fluid was detected in the posterior cul-de-sac (Fig. 4). The formation on the right was consistent with an ectopic pregnancy in the fallopian tube corresponding to the gestational age of 5 weeks and 6 days. M -mode and color Doppler revealed two fetal heart tones: The 1st embryo had 97 beats per minute and the 2nd 86 beats per minute (Fig. 5). Color Doppler enabled visualization of randomly dispersed tubal arteries and heart activity of both embryos (Figs 6 and 7).

Laparoscopy was then performed removing the right fallopian tube. The specimen submitted consisted of grossly identified fallopian tube and fimbria that measured $6.5 \times 1.5 \times$ $1.5 \mathrm{~cm}$. When cut longitudinally, the tube reveal ed two close together nodules composed of spongiose, focally hemorrhagic tissue, largest measuring $1.5 \mathrm{~cm}$ in diameter and the smallest measuring $0.8 \mathrm{~cm}$ in diameter. 


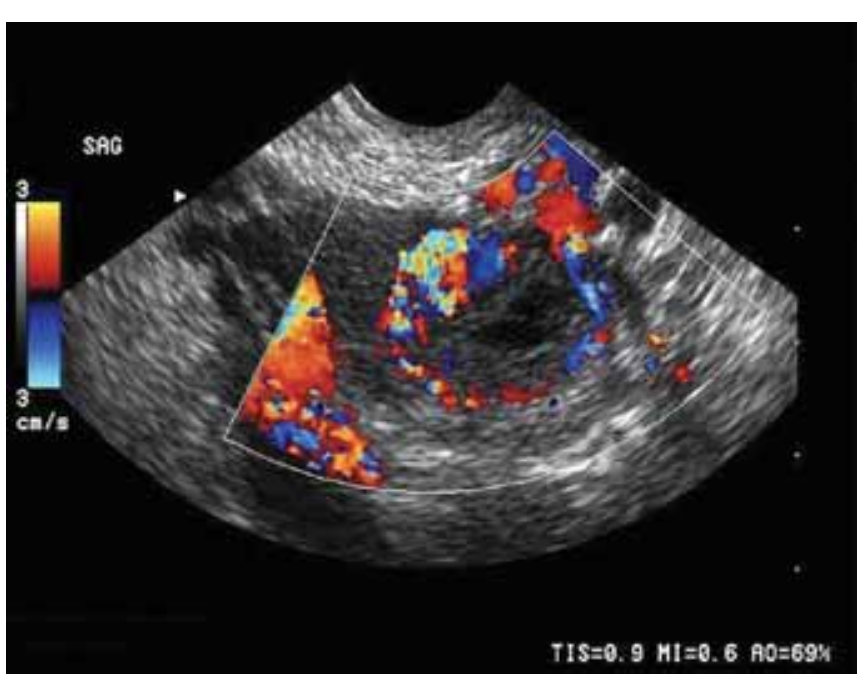

Fig. 6: Transvaginal color Doppler of ectopic pregnancy. Color Doppler facilitates visualization of randomly dispersed tubal arteries indicating prominent trophoblastic vitality and invasiveness

The patient was discharged home on postoperative day 1 , with an uneventful hospital stay. The patient's beta hCG level was followed on outpatient's basis until it was undetectable.

\section{DISCUSSION}

A though the mortality associated with ectopic pregnancy has steadily decreased due to advancements made in both medical and surgical technology, the incidence of ectopic pregnancies has increased from $0.5 \%$ in 1970 to $2 \%$ today due to the increasing rate of STDs and ART, 6,7

Currently, there are approximately 100 cases published in the literature diagnosing unilateral twin ectopic pregnancies preoperatively and only 13 diagnosed cases which have documented fetal heart motion in live twin gestation. 4,13

$M$ edical management of ectopic pregnancies includes the use of methotrexate. This was first utilized by Tanaka et al to treat an interstitial pregnancy in 1982 and has since become a popular outpatient treatment. ${ }^{8}$ C riteria with the greatest success for this approach include a beta hCG level of $<2500 \mathrm{mU} / \mathrm{ml}^{9}$ and an ectopic size of $<3.5 \mathrm{~cm} .{ }^{10} \mathrm{~A}$ s recently shown in a study by Lipscomb et al, when using methotrexate as therapy, the size or volume of the conceptus is an important factor, how ever, the single most important factor in determining the success of single-dose methotrexate is the beta hC G concentration. ${ }^{14} \mathrm{~T}$ his was due to the fact that a high beta hCG was an indicator of a still growing and developing conceptus. A Iso, the presence of fetal cardiac activity has shown a decrease in the success of single-dose methotrexate therapy, likely due to the more advanced stage in gestation. However, if detected at an early stage in a hemodynamically stable w oman, methotrexate therapy can be effective. This was recently shown in a similar case by A rikan et al. ${ }^{16}$

Surgical management preferred in the U nited States is the laparoscopic approach. These techniques include sal pingostomy and salpingectomy. Salpingostomy is used to remove a

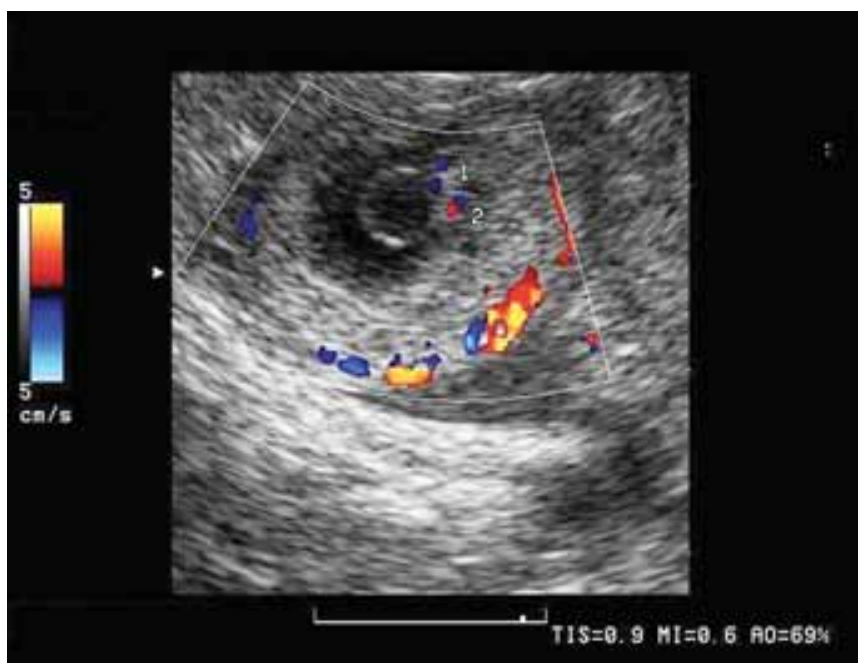

Fig. 7: Prominent blood flow signals indicate vital trophoblast. In addition to peritrophoblastic flow, color Doppler can depict cardiac activity of both embryos ( 1 and 2 )

pregnancy $<2 \mathrm{~cm}$ in length and located in the distal third of the fallopian tube. This approach is used when fertility preservation is desired in the near future. Salpingectomy is a procedure that involves tubal resection and is an approach that can be used when the tube is ruptured or unruptured.

In a Cochrane review of 35 studies completed on the success of various managements of ectopic pregnancies, it has been shown that with long-term follow-up with a medical versus surgical approach, there is no significant difference in either treatment regimen. There was also no evidence of difference in future intrauterine pregnancy rates. ${ }^{11} \mathrm{H}$ owever, a study completed by M onika et al has shown that of patients who have IV F performed, there is an $8 \%$ increased rate of repeated tubal ectopic pregnancy when a prior tubal ectopic pregnancy has occurred. ${ }^{12}$ A nother conservative approach to the treatment of tubal ectopic pregnancy described by Fernandez et al is ultrasound-guided injection of methotrexate directly in the affected tube. ${ }^{15,16}$

The ultrasound machine with color Doppler facility is an excellent guide to search for blood flow signals within the entire pelvis. The color flow pattern associated with an ectopic pregnancy is variable. It usually presents as randomly dispersed multiple small vessels within the adnexa, showing high-velocity and low impedance signals ( $R$ I 0.38 to 0.45 ), which are clearly separated from the ovarian tissue and corpus luteum. The sensitivity of the transvaginal color and pulsed Doppler in the diagnosis of an ectopic pregnancy has been reported by several studies and ranges from 73 to $96 \%$, and the specificity ranges from 87 to $100 \% .{ }^{17,18}$ Demonstration of a 'ring of fire' is due to the very high speed of the peritrophoblastic blood flow and low impedance. It should be stressed that patients with tubal abortion demonstrate significantly higher vascular impedance of peritrophoblastic flow $(\mathrm{RI}>0.60)$ and less prominent color signals.

$V$ isualization of the ipsilateral corpus luteum blood flow may aid in the diagnosis of an ectopic pregnancy, since in 
majority of patients with a proven ectopic pregnancy, luteal flow is detected on the same side as the ectopic pregnancy.

The main diagnostic importance of transvaginal color and pulsed Doppler is in differentiating the nature of a nonspecific adnexal mass. ${ }^{19,20}$ As with other diagnostic methods, transvaginal color and pulsed Doppler studies include both, false-positive and false-negative findings. A false-positive diagnosis arises predominantly from the corpus luteum, but in exceptional cases some adnexal lesions may mimic an ectopic pregnancy. A false-negative result may arise from technical inadequacy, lack of operator's experience or the patients' non-compliance. The other possibility of a faulty diagnosis is a non-vascularized ectopic gestation, as these are associated with low beta hCG values.

A fter implantation in the mucosa of endosalpinx, the lamina propria and then the muscularis of the oviduct, the blastocyst grows mainly between the lumen of the tube and its peritoneal covering. ${ }^{19} \mathrm{G}$ rowth occurs both parallel to the long axis of the tube and circumferentially around it. A s the trophoblast invades surrounding vessels, intensive blood flow and/or intraperitoneal bleeding may occur. The intensive ring of vascular signals could be a criterion for viability of an ectopic pregnancy that can be determined rapidly and easily and seems to be independent of beta hCG values. In patients undergoing a conservative treatment, this method could provide an aid in addition to beta hCG values for supervising the efficiency of therapy. This way duration of the hospitalization could be shortened, the patient's uncertainty diminished and the cost of the treatment reduced. In cases of persistent high beta hCG levels after operative removal of the ectopic pregnancy, color Doppler sonography can provide evidence for the presence of viable trophoblast remnants. On the contrary, in asymptomatic patients with hypoperfused and/or avascular ectopic gestational sac and decreased values of beta hCG, expectant treatment can be established. ${ }^{19}$

M ore recently, it has been reported that three-dimensional ultrasound may aid in the management of ectopic pregnancy. Three-dimensional ultrasound with color and/or power Doppler has proven useful in both the initial diagnosis and follow-up of ectopic pregnancies especially with assisted reproduction treatment and embryo transfer ${ }^{21}$ Preliminary data of other authors suggested that 3D sonography is an effective procedure for early diagnosis of ectopic pregnancy in asymptomatic patients before 6 weeks of amenorrhea. The possible use of 3D power Doppler is the monitoring of the vascularity of an ectopic pregnancy. The hypoperfusion, quantified by indices of vascularity (VI) and flow (FI), could indicate that the ectopic pregnancy is spontaneously being resolved, and that laparoscopy should be postponed. This way, the conservative approach to an ectopic pregnancy would rely on more precise and easily obtainable data. In case of hyperperfusion, the patients should be subjected to laparoscopy or medical treatment immediately.

Shih et al ${ }^{22}$ described the use of 3D color/power angiography in two cases in which an arteriovenous malformation of the mesosalpinx was diagnosed following involution of an anembryonic ectopic gestation. The diagnosis of arteriovenous malformations has traditionally been made by arteriography. Recently, it has also been diagnosed by noninvasive methods such as contrast enhanced CT, MRI and color Doppler ultrasound. The advantage of 3D reconstruction of color/power angiography images is a better spatial and anatomic orientation and a quick demonstration of the vessels, usually within one minute, especially in the areas where complex structures are present. Therefore, unlike M RI, digital subtraction angiography or contrast-enhanced CT, 3D color/power angiography allow the physician to examine vascular anatomy immediately and without radiation exposure.

Even though the exact role of 3D ultrasound in the pathology of early pregnancy is yet to be established, promising results in al ready published papers are encouraging. ${ }^{23} \mathrm{U}$ nlimited numbers of sections are easily obtained without the need for excessive manipulation of the probe. A dditional progress has been made, owing to the permanent possibility or repeated analysis of previous stored 3D volumes and Cartesian elimination of surrounding structures and artifacts. A three-dimensional reconstruction of stored images without any degradation is the most impressive benefit of 3D scanning.

U Itimately, with the advancement of both earlier detection of beta hCG levels at earlier gestational age, and the use of high-resolution 2D and 3D ultrasound coupled with color and power Doppler imaging for diagnosis and treatment of tubal gestation, there should be an increase in subsequent fertility in women affected by ectopic pregnancies.

\section{REFERENCES}

1. A merican College of Obstetrics and Gynecologist: M edical management of ectopic pregnancy. Practice Bulletin 2008;94.

2. Bouyer J, Coste J, Shojaei T, et al. Risk factors for ectopic pregnancy: A comprehensive analysis based on a large casecontrol, population-based study in France. A m J Epidemiol 2003;157:185.

3. A brams RA, Kanter AE. Bilateral simultaneous extrauterine pregnancy. A m J Obstetrics and Gynecology 1948;56(6):11981200.

4. Eddib A, Olawriye A, Withiam-L eitch M, R odgers B, Y eh. Liver twintubal ectopic pregnancy. Int] Gynecol O bstet2006;93:154-55.

5. De Ott. A case of unilateral tubal twin gestation. Annals de Gynécolgie et d'O bstétrique 1891;36;304.

6. Dammann G, M urphy L, Ellis J. Twin heterotrophic pregnancy after assisted reproduction. J Reprod M ed 2000;47(3):246-48.

7. Géraed HC, B ranigan PJ, B alsara GR, Heath C, M inassian SS, Hudson AP. Viability of Chlamydia trachomatis in fallopian tubes of patients with ectopic pregnancy. Fertility and Sterility 1998;70(5):945-48.

8. Tanaka T, Hayashi H, K utsuzawa T, et al. Treatment of interstitial ectopic pregnancy with methotrexate: Report of a successful case. Fertility and Sterility 1982;37:851.

9. Lehner R, K ucera E, Jirecek S, E garter C, Husslein P. Ectopic pregnancy. A rch Gynecol O bstet 2000;263:87-92.

10. Lipscomb GH, M cCord ML, Stovall TG, et al. Predictors of successful methotrexate treatment in women with tubal ectopic pregnancies. N Engl J Med 1999;341:4.

11. Hajenius, Petra J, Mol, Femke, Mol, Ben Willem J, et al. Interventions for tubal ectopic pregnancy. Cochrane Database of Systematic R eviews 2009.

12. W eigert M onika, Gruber Diego, Pernicka Elisabeth, B auer Peter, Feichtinger Wilfried. Previous tubal ectopic pregnancy raises the incidence of repeated ectopic pregnancies in in vitro fertilization-embryo transfer patients. J A ssist Reprod Genet 2009;26(1):13-17. 
13. Birte Summa, Ivo M einhold, Dirk O. Bauerschlag et al. Early detection of twin tubal pregnancy by Doppler sonography allows fertility-conserving laparoscopic surgery. A rch Gynecol O bstet 2009;279:87-90.

14. Lipscomb, Gary, M cCord, M arian, et al. Predictors of success of methotrexate treatment in women with tubal ectopic pregnancies. NEJM 1999;341:1974-78.

15. Ferbabdez $\mathrm{H}$, Pauthier $\mathrm{S}$, et al. U Itrasound-guided injection of methotrexate versus laparoscopic salpingotomy in ectopic pregnancy. Fertility and Sterility 1999;1:25-29.

16. M orlock RJ, Lafata JE, Eisenstein D. Cost-effectiveness of single-dose methotrexate compared with laparoscopic treatment of ectopic pregnancy. Obstet Gynecol 2000;95:407-12.

17. Kupesic S, Kurjak A. Color Doppler assessment of ectopic pregnancy. In: Kurjak A, Kupesic S (Eds). An A tlas of Transvaginal Color Doppler. London: Parthenon Publishing, 2000:137-47.

18. Kurjak A, Zalud I, Shulman H. Ectopic pregnancy: Transvaginal color Doppler of trophoblastic flow in questionable adnexa. J Ultrasound M ed 1991;10:685-89.
19. K upesic Plavsic S, Zafar N, Honemeyer U . Ectopic pregnancy: Diagnosing and treating the challenge. In: Donald School Textbook of U Itrasound in Obstetrics and Gynecology, K urjak A , Chervenak F (Eds). Jaypee B rothers M edical Publishers LTD; L ondon, New Delhi, Panama City, St. L ouis; 130-48.

20. K upesic S, K urjak A, A ksamija A. Ectopic pregnancy: A dvances in early diagnosis made by color Doppler and three-dimensional ultrasound. Ultrasound Rev O bstet Gynecol 2002;2:1-16.

21. Raine Fenning N, Fleicher AC. Clarifying the role of threedimensional transvaginal sonography in reproductive medicine: A nevidence-based appraisal.J ExpC linA ssistReprod 2005;2:10.

22. Shih JC, Shyu M K, Cheng WF, Lee CN, Jou HJ, Wang RM, $\mathrm{H}$ seih FJ . A rteriovenous malformation of mesosal pinx associated with a vanishing ectopic pregnancy: Diagnosis with threedimensional color power angiography. Ultrasound Obstet Gynecol 1999;13:63-66.

23. Harika G, Gabriel R, Carre-Pigeon F, A lemany L, Q uereux C, $W$ ahl P. Primary application of three-dimensional ultrasonography to early diagnosis of ectopic pregnancy. Eur J Obstet Gynecol Reprod Biol 1995;60:117-20.

After you have reviewed the case report, discussion and images, take a quiz to test your knowledge and competency:

\section{The most common site of ectopic pregnancy is the:}
a. Ovary
b. Cervix
c. Fallopian Tube
d. A bdomen
e. Cornual region of the uterus

2. Which of the following is risk factor for an ectopic pregnancy?
a. A history of sexually transmitted disease
b. A history of assisted reproduction techniques
c. Prior tubal gestation
d. Smoking
e. All of the above

3. The classic symptoms of ectopic pregnancy include one of the following except:
a. A menorrhea
b. A bnormal genital tract bleeding
c. A dnexal tenderness
d. A dnexal mass
e. Syncope

4. The discriminatory zone beyond which a gestational sac is visualized in the uterus with a transvaginal sonography is:
a. $500 \mathrm{mlU} / \mathrm{ml}$

b. $1.500 \mathrm{mlU} / \mathrm{ml}$
c. $2.500 \mathrm{mlU} / \mathrm{ml}$
d. $3.500 \mathrm{mlU} / \mathrm{ml}$
e. $4.500 \mathrm{mlU} / \mathrm{ml}$

5. The most common sonographic sign associated with an ectopic pregnancy is:
a. A complex adnexal mass
b. A pseudogestational sac
c. An echogenic ectopic ring
d. F ree fluid in the posterior cul-de-sac
e. Hyperechogenic endometrium

6. T win ectopic pregnancy is estimated to occur in:
a. 1:50.000 ectopic pregnancies
b. 1:100.000 ectopic pregnancies
c. 1:200.000 ectopic pregnancies
d. 1:125.000 ectopic pregnancies
e. 1:225.000 ectopic pregnancies

\section{Demonstration of 'ring of fire' in ectopic pregnancy indicates:}
a. High speed of the peritrophoblastic blood
b. Low impedance in tubal arteries
c. Trophoblastic vitality
d. Trophoblastic invasiveness
e. All of the above

\section{Correct Answers:}

$1-C$

$\begin{array}{lllllll}\text { B } & 5 & \text { C } & 6 & \text { D } & 7 & \text { E }\end{array}$

